\title{
Gonadal-sparing total body irradiation with the use of helical tomotherapy for nonmalignant indications
}

\author{
Khaled Dibs ${ }^{1 *}$, Austin J. Sim² ${ }^{2 *}$ José A. Peñagaricano², Kujtim Latifi ${ }^{2}$ Genevieve A. Garcia ${ }^{2}$, Julia A. Peters ${ }^{2}$, \\ Michael L. Nieder ${ }^{3}$, Sungjune Kim², Timothy J. Robinson ${ }^{2,4}$ \\ ${ }^{1}$ Department of Radiation Oncology, The Ohio State University, Columbus OH, United States \\ ${ }^{2}$ Department of Radiation Oncology, H. Lee Moffit Cancer Center and Research Institute, Tampa, FL, United States \\ ${ }^{3}$ Department of Blood and Marrow Transplant and Cellular Immunotherapy, H. Lee Moffitt Cancer Center and Research Institute, \\ Tampa, FL, United States \\ ${ }^{4}$ Department of Biostatistics and Bioinformatics, H. Lee Moffit Cancer Center and Research Institute, Tampa, FL, United States \\ ${ }^{\star}$ Co-first authors
}

\begin{abstract}
Background: The aim was to demonstrate the feasibility and technique of gonadal sparing total body irradiation (TBI) with helical tomotherapy. Total body irradiation is a common part of the conditioning regimen prior to allogeneic stem cell transplantation. Shielding or dose-reduction to the gonads is often desired to preserve fertility, particularly in young patients undergoing transplant for non-malignant indications. Helical tomotherapy $(\mathrm{HT})$ has been shown to be superior to traditional TBI delivery for organ at risk (OAR) doses and dose homogeneity.

Materials and methods: We present two representative cases (one male and one female) to illustrate the feasibility of this technique, each of whom received $3 \mathrm{G} y$ in a single fraction prior to allogeneic stem cell transplant for benign indications. The planning target volume (PTV) included the whole body with a subtraction of OARs including the lungs, heart, and brain (each contracted by $1 \mathrm{~cm}$ ) as well as the gonads (testicles expanded by $5 \mathrm{~cm}$ and ovaries expanded by $0.5 \mathrm{~cm}$ ).

Results: For the male patient we achieved a homogeneity index of 1.35 with a maximum and median planned dose to the testes of $0.53 \mathrm{~Gy}$ and $0.35 \mathrm{~Gy}$, respectively. In-vivo dosimetry demonstrated an actual received dose of $0.48 \mathrm{~Gy}$. For the female patient we achieved a homogeneity index of 1.13 with a maximum and median planned dose to the ovaries of $1.66 \mathrm{~Gy}$ and $0.86 \mathrm{~Gy}$, respectively.

Conclusion: Gonadal sparing TBl is feasible and deliverable using HT in patients with non-malignant diseases requiring TBI as part of a pre-stem cell transplant conditioning regimen.
\end{abstract}

Key words: gonadal sparing; helical tomotherapy; marrow transplantation

Rep Pract Oncol Radiother 2021;26(1):153-158

\section{Introduction}

Total body irradiation (TBI) is commonly used in patients undergoing allogenic stem cell transplantation for both malignant and benign processes. Both traditional TBI approaches (opposed lateral and $\mathrm{AP} / \mathrm{PA}$ ) use extended source to skin dis- tances (SSDs), custom compensators, blocks, and beam spoilers to ensure adequate skin dose [1]. However, traditional linear accelerator-based delivery has several limitations, including dose-limiting toxicity $[2,3]$. For men, attempts to use a "clamshell" testicular shield may increase dose due to increased electron scatter [4]. More recently, volu-

Address for correspondence: Timothy J. Robinson, MD, PhD, Department of Radiation Oncology, H. Lee Moffitt Cancer Center and Research Institute, 12902 USF Magnolia Dr., Tampa, FL 22612, United States, tel: 813-745-4673; e-mail: Timothy.Robinson@moffitt.org 
metric modulated arc therapy (VMAT) has been used for better dose distributions on standard linear accelerators, but this method can become overly complex with multi-isocentric setups and extended planning and delivery times [5]. The advantages of helical tomotherapy (HT)-based TBI include radial delivery of dose [6], creating better dose distribution, increased conformity and homogeneity, and image guidance, without the need for the above modifiers or compacted patient positioning (lateral technique) [7].

An unmet need in TBI is the ability to spare dose to the gonads, which can be technically challenging in full grown adolescents or young adults. Radiotherapy can cause temporary azoospermia at low doses and recovery of spermatogenesis may take up to 30 months after a single dose of 2-3 Gy and up to 5 years with a dose of $4 \mathrm{~Gy}$ [8]. Although azoospermia can become permanent after 6-8 Gy in 2-Gy fractions [9], the $\mathrm{LD}_{50}$ of the human oocyte is $<2$ Gy, potentially leading to permanent sterility in women [10]. Based on previous reports on HT-TBI feasibility and fertility preservation needs, we used HT in two young adults undergoing $3 \mathrm{~Gy}$ $\mathrm{TBI}$ in preparation for allogeneic transplant for the treatment of severe sickle cell anemia.

\section{Materials and methods}

A 20-year old man with severe sickle beta-zero thalassemia and a 32-year old woman with severe sickle cell anemia both underwent conditioning with alemtuzumab and a single fraction of TBI to $3 \mathrm{~Gy}$ prior to matched allogeneic transplants. $\mathrm{Pa}$ tient height precluded AP/PA TBI with lead or ceramide-block shielding. We, therefore, investigated the ability to deliver TBI using HT for gonadal sparing using a TomoTherapy $\mathrm{H}$ unit (Accuray, Inc., Sunnyvale, CA).

\section{CT simulation}

Immobilization was achieved using a vacuum cushion (BlueBAG BodyFIX, Elekta AB, Stockholm, Sweden) and a custom thermoplastic mask in a supine position with arms by the side. An initial CT scan was completed head-first from the vertex through the mid femur and a second scan was completed feet-first for the remainder of the lower extremities, both with a slice thickness of $3 \mathrm{~mm}$. Due to the field length limitations of our
HT unit (up to $160 \mathrm{~cm}$ ), two image sets were used to generate two HT plans, with a match line delineated at the mid femur with radiographically opaque wire well below the level of the genitals to avoid hotspots.

\section{Organs at risk (OARs) and target volumes}

The brain, lungs, heart, kidneys, and gonads were delineated as OARs. Avoidance structures of each of these organs were created as follows: the brain, lungs, and heart were each contracted by $1 \mathrm{~cm}$, the kidneys were unchanged, the testicles were expanded by $5 \mathrm{~cm}$, and the ovaries were expanded by $0.5 \mathrm{~cm}$ and contracted out of the bony pelvis $+1 \mathrm{~cm}$ (Fig. 1A, 2A). The lungs and heart were contracted to ensure the rib cage remained within the treatment target volume during normal respiratory motion. We did not include the liver as an avoidance structure, given the potential for Kupffer cells or other immune infiltrates. However, the exclusion of the liver from target volumes would not be unreasonable.

The clinical target volume (CTV) was defined as the whole body minus the gonadal avoidance structures, lungs, heart, and brain. This was then expanded by $1 \mathrm{~cm}$ and the avoidance structures (brain $-1 \mathrm{~cm}$, lungs/heart $-1 \mathrm{~cm}$, kidneys, testes $+5 \mathrm{~cm}$, ovaries $+0.5 \mathrm{~cm}$ ) were subtracted to create the planning target volume (PTV).

\section{TBI planning}

An HT plan for TBI was generated for these patients with $96 \%$ of the PTV being covered by the prescription dose of 3 Gy using a field width of 5 $\mathrm{cm}$ and a standard grid size (Fig. 1B, 2B). Planning proceeded as described by Hui et al. [11]. Briefly, TERMA (total energy released per unit mass) optimizations were performed to find the lowest modulation factor (MF) and the loosest pitch capable of maintaining the dose-volume histogram characteristics of a plan with a high MF and tight pitch while achieving a reasonable total treatment time [11]. This resulted in an MF and a pitch of 2 and 0.330, respectively, for the first plan and a pitch of 2 and MF of 0.303 for the second. Approximately $15-20$ optimizations were needed to view the result of the constraint adjustment, which took 45-60 minutes. The same jaw width, pitch, and MF were used for the lower extremity plans. 

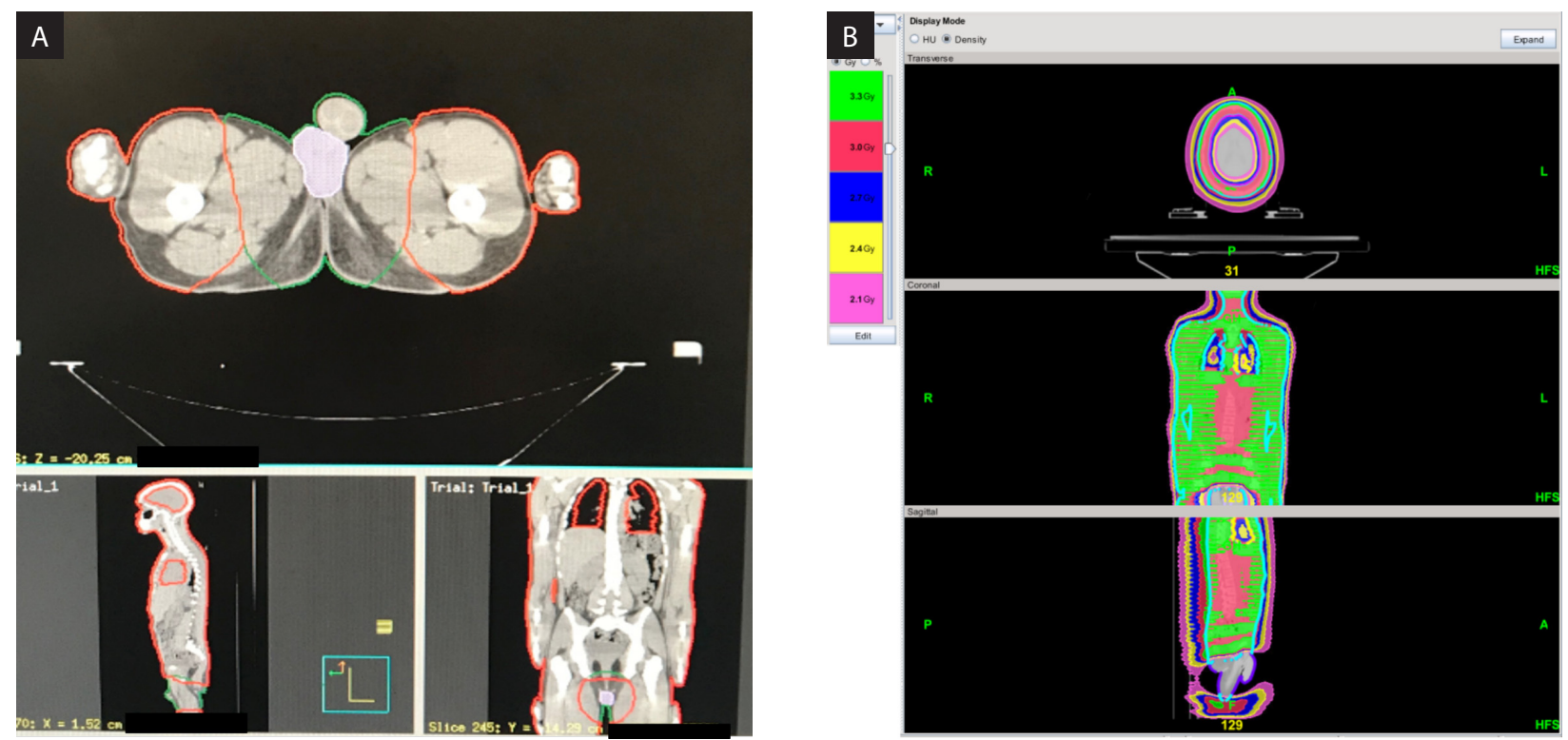

Figure 1. A. Testicular avoidance structure. B. Isodose distribution. 3 Gy was prescribed to $95 \%$ of the planning target volume (PTV)
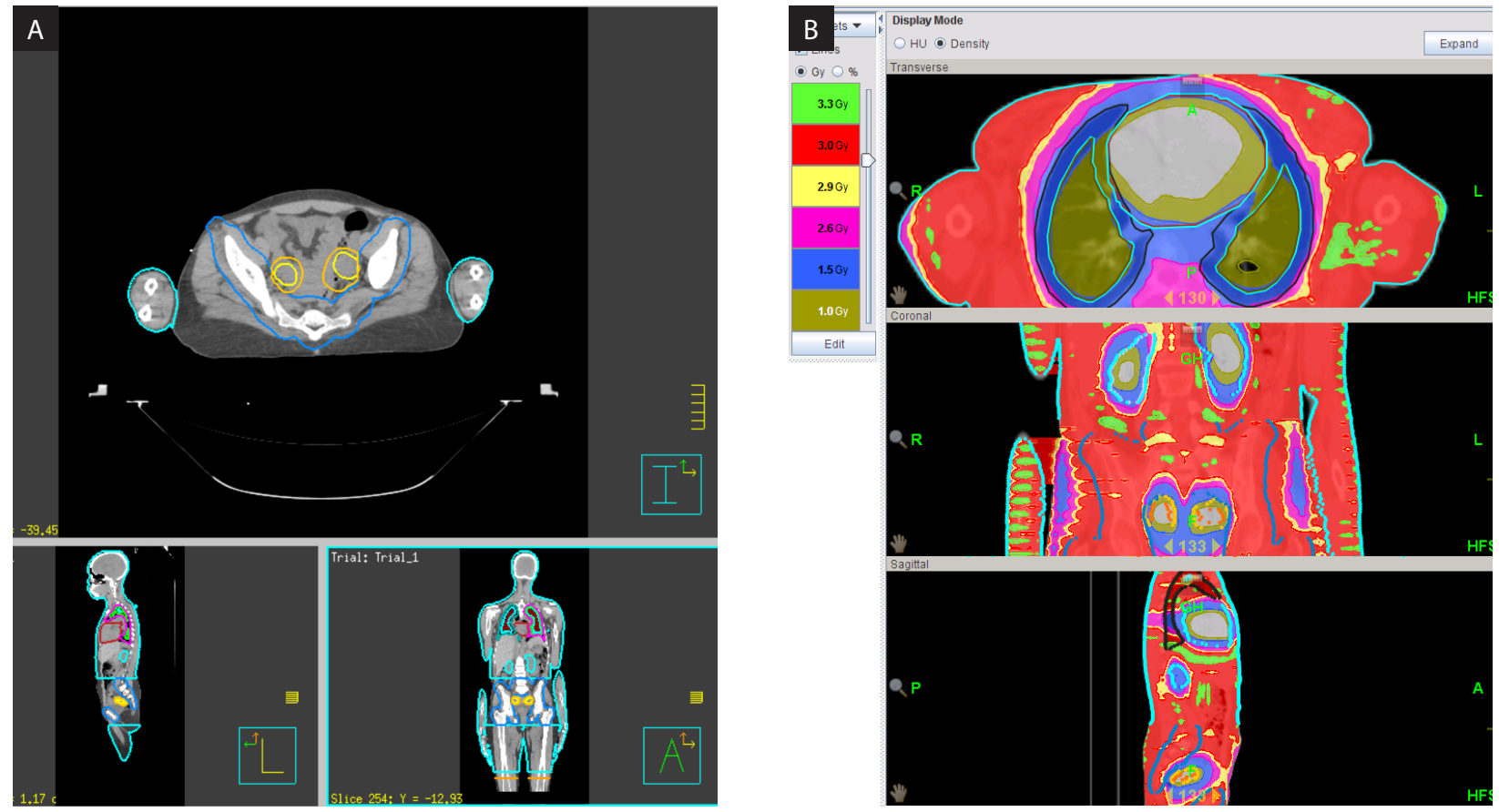

Figure 2. A. Ovarian avoidance structure. B. Isodose distribution. 3 Gy was prescribed to $95 \%$ of the planning target volume (PTV).

\section{Quality assurance (QA)}

For QA, a 3D diode array cylindrical phantom was imaged (Delta4, Scandidos Uppsala, Sweden) [12] on the CT simulator scanner and imported into the TomoTherapy planning station. The patient treatment plans were transferred onto the phantom image and dose distribution based on the phantom material and geometry was calculated. The gamma $(3 \%, 3 \mathrm{~mm})$ passing rate criteria between the calculated and measured plans was set at $90 \%$. Because of the size of the PTVs, two QA plans were applied to the phantom (head first and feet first). 


\section{Treatment delivery}

Prior to treatment delivery, four megavoltage computed tomography (MVCT) scans were obtained in the treatment position in the coarse mode (head, thorax, pelvis, feet). Automatic kVCT to MVCT fusion was performed on the basis of these regions of interest. Manual fusion was performed to improve the registration accuracy by verifying axial, coronal and sagittal images. This entire process took 15-20 minutes. Once the registration was approved, the couch was positioned to the determined registration coordinates and treatment was delivered.

\section{Results}

For the first case, the overall beam-on time was approximately 43 minutes. The maximum and median doses to the testes were $0.53 \mathrm{~Gy}$ and $0.35 \mathrm{~Gy}$, respectively (Tab. 1). The in-vivo dosimetry was calculated using the NanoDot ${ }^{\mathrm{TM}}$ (Landauer, Glenwood, IL) and confirmed that the testes received $0.48 \mathrm{~Gy}$. Based on the in-vivo dosimetry, the difference between planned and delivered doses ranged from $-17.8 \%$ to $14.6 \%$ (Tab. 2). The second case achieved maximum and median doses to the ovaries of 1.66 Gy and 0.86 Gy, respectively (Tab. 1). In-vivo dosimetry of the ovaries was not feasible, but the difference between planned and delivered doses to other areas ranged from $-26.1 \%$ to $43.6 \%$ (Tab. 2).

Both patients successfully underwent bone marrow transplant after completion of the conditioning regimen. They were both discharged after an uneventful hospital course. The first patient's most recent follow-up was 25 months after transplant and unremarkable. The second patient's most recent follow-up was 3 months after transplant and unremarkable as well.

\section{Discussion}

To the authors' knowledge, we describe the first technical report of using HT-TBI to provide gonadal sparing in full-grown adults for non-malignant conditions. With HT, there is no need to use heavy blocks, beam spoilers, an extended source to skin distance, or uncomfortable patient positioning. The ability to reduce the delivered dose to the testes to $0.48 \mathrm{~Gy}$ will nearly eliminate the risk of long-term azoospermia [9] and maintaining the maximum delivered dose to the ovaries to 1.66 Gy will greatly reduce the risk of premature ovarian failure [10]. Conversely, when clinical scenarios require boosting the gonads [13], HT could easily be used to deliver a simultaneous integrated boost. This method lies within a spectrum of techniques between traditional TBI to more focused total marrow irradiation (TMI) and total marrow and lymph node irradiation (TMLI). Although the latter hold promise to reduce toxicity and dose escalation targets, availability and implementation are quite heterogeneous [14] and may involve higher dose rates, potentially increasing toxicity, or high marrow doses, increasing the risk of nonengraftment [13]. While this method conceptually shifts toward TMI, the continued difference in targets serves to distinguish the two methods.

Table 1. Achieved doses for organs at risk based off of the planned dosimetry

\begin{tabular}{|c|c|c|c|c|c|c|}
\hline \multirow{3}{*}{ OAR } & \multicolumn{6}{|c|}{ Planned dosimetry } \\
\hline & \multicolumn{3}{|c|}{ Case 1} & \multicolumn{3}{|c|}{ Case 2} \\
\hline & Max dose (Gy) & Median dose (Gy) & Mean dose (Gy) & Max dose (Gy) & Median dose (Gy) & Mean dose (Gy) \\
\hline Lungs & 3.58 & 3.14 & 3.06 & 3.39 & 2.28 & 2.22 \\
\hline Heart & 3.53 & 2.82 & 2.8 & 3.04 & 1.08 & 1.22 \\
\hline Testicles & 0.53 & 0.35 & 0.36 & $\mathrm{~N} / \mathrm{A}$ & $\mathrm{N} / \mathrm{A}$ & $\mathrm{N} / \mathrm{A}$ \\
\hline Testicles $+5 \mathrm{~cm}$ & 3.09 & 0.55 & 0.69 & $\mathrm{~N} / \mathrm{A}$ & $\mathrm{N} / \mathrm{A}$ & N/A \\
\hline Ovary & $\mathrm{N} / \mathrm{A}$ & N/A & N/A & 1.66 & 0.86 & 0.89 \\
\hline Adnexa & $\mathrm{N} / \mathrm{A}$ & N/A & $\mathrm{N} / \mathrm{A}$ & 2.48 & 0.95 & 1.03 \\
\hline Right kidney & 3.48 & 3.26 & 3.27 & 2.94 & 1.15 & 1.37 \\
\hline Left kidney & 3.39 & 3.22 & 3.22 & 2.93 & 1.12 & 1.25 \\
\hline
\end{tabular}

OAR — organ at risk; N/A - non available 
Table 2. Measured doses actually delivered based off of in vivo dosimetry obtained with NanoDot ${ }^{\mathrm{TM}}$ when compared to the target dose

\begin{tabular}{|c|c|c|c|c|c|c|}
\hline \multirow{3}{*}{ Location } & \multicolumn{6}{|c|}{ In-vivo dosimetry } \\
\hline & \multicolumn{3}{|c|}{ Case 1} & \multicolumn{3}{|c|}{ Case 2} \\
\hline & $\begin{array}{l}\text { Target dose } \\
\text { (cGy) }\end{array}$ & $\begin{array}{l}\text { Measured dose } \\
\text { (cGy) }\end{array}$ & $\%$ difference & $\begin{array}{l}\text { Target dose } \\
\text { (cGy) }\end{array}$ & $\begin{array}{l}\text { Measured dose } \\
\text { (cGy) }\end{array}$ & $\%$ difference \\
\hline Umbilicus & 300 & 310.3 & 3.4 & 300 & 291.6 & -2.8 \\
\hline Head & 300 & 316.0 & 5.2 & 300 & 331.2 & 10.4 \\
\hline Right neck & 300 & 272.8 & -9.5 & & & \\
\hline Right shoulder & 300 & 313.7 & 4.5 & 300 & 321.7 & -2.5 \\
\hline Mid mediastinum & 300 & 275.3 & -8.6 & & & \\
\hline Right breast & 300 & 309.9 & 3.2 & & & \\
\hline Left breast & 300 & 307.1 & 2.3 & 300 & 346.3 & 4.9 \\
\hline Mid thigh (overlap) & 300 & 344.7 & 13.9 & & & \\
\hline R thigh (overlap) & & & & 300 & 430.9 & 43.6 \\
\hline L thigh (overlap) & & & & 300 & 221.7 & -26.1 \\
\hline Right foot & 300 & 251.0 & -17.8 & 300 & 331.2 & 10.4 \\
\hline Left hand & 300 & 347.1 & 14.6 & 300 & 306.2 & 3.1 \\
\hline Testicles & & 48.0 & & & & \\
\hline Beam on time & & 43 minutes & & & 41 minutes & \\
\hline Conformity index & & 0.96 & & & 0.96 & \\
\hline $\begin{array}{l}\text { Homogeneity } \\
\text { index }\end{array}$ & & 1.35 & & & 1.13 & \\
\hline
\end{tabular}

Total body irradiation continues to be a critical tool in bone marrow transplantation to decrease tumor burden in malignant diseases and to suppress the recipient's immune system to improve the likelihood of graft rejection [15]. This report also demonstrates the homogeneity and image guidance abilities of HT-TBI, in contrast to classic TBI with dose variations of up to $50 \%$ due to thickness difference at different level of the body [16]. In our representative cases, the homogeneity indices were 1.35 and 1.13 , respectively.

\section{Conclusion}

In this Technical Report, we demonstrate the feasibility and potential benefits of using HT-TBI over traditional methods. Future research is warranted to explore potential benefits of targeted TBI, both in the optimal approach for transplantation and special circumstances as described in this report.

\section{Conflicts of interest}

The author and co-authors declare no conflicts of interest.

\section{Funding}

No funding was procured or used in preparation of this manuscript.

\section{Clinical trial information}

N/A.

None.

\section{Acknowledgements}

All data generated and analyzed during this study are included in this published article.

Part of this work was presented at the American College of Radiation Oncology 2020 Annual Meeting, Ft. Lauderdale, FL, 2020 Feb 26-29.

\section{References}

1. Litoborska J, Piotrowski T, Jodda A, et al. Evolution of treatment planning and dose delivery methods during radiotherapy for patients undergoing bone marrow transplantation: a review. Nukleonika. 2020; 65(1): 19-30, doi: 10.2478/nuka-2020-0003.

2. Stephens SJ, Thomas S, Rizzieri DA, et al. Myeloablative conditioning with total body irradiation for AML: Balanc- 
ing survival and pulmonary toxicity. Adv Radiat Oncol. 2016; 1(4): 272-280, doi: 10.1016/j.adro.2016.07.001, indexed in Pubmed: 28740897.

3. Borg $M$, Hughes $T$, Horvath $N$, et al. Renal toxicity after total body irradiation. Int J Radiat Oncol Biol Phys. 2002; 54(4): 1165-1173, doi: 10.1016/s0360-3016(02)03039-0, indexed in Pubmed: 12419445.

4. Sanghvi PR, Kaurin DGL, McDonald TL, et al. Testicular shielding in low-dose total body irradiation. Bone Marrow Transplant. 2007; 39(4): 247-248, doi: 10.1038/ sj.bmt.1705574, indexed in Pubmed: 17290280.

5. Springer A, Hammer J, Winkler E, et al. Total body irradiation with volumetric modulated arc therapy: Dosimetric data and first clinical experience. Radiat Oncol. 2016; 11: 46, doi: 10.1186/s13014-016-0625-7, indexed in Pubmed: 27000180.

6. Peñagarícano J, Moros E, Corry P, et al. Pediatric craniospinal axis irradiation with helical tomotherapy: patient outcome and lack of acute pulmonary toxicity. Int J Radiat Oncol Biol Phys. 2009; 75(4): 1155-1161, doi: 10.1016/j. ijrobp.2008.12.083, indexed in Pubmed: 19467796.

7. Peñagarícano JA, Chao M, Van Rhee F, et al. Clinical feasibility of TBI with helical tomotherapy. Bone Marrow Transplant. 2011; 46(7): 929-935, doi: 10.1038/bmt.2010.237, indexed in Pubmed: 20935684.

8. Howell SJ, Shalet SM. Spermatogenesis after cancer treatment: damage and recovery. J Natl Cancer Inst Monogr. 2005(34): 12-17, doi: 10.1093/jncimonographs/lgi003, indexed in Pubmed: 15784814.

9. Hall EJ, Giaccia AJ. Clinical response of normal tissue. In: Hall EJ, Giaccia AJ. ed. Radiobiology for the Radiologist. Seventh ed. Wiliams \& Wilkins, Philadelphia 2012.
10. Wallace WHB, Thomson AB, Kelsey TW. The radiosensitivity of the human oocyte. Hum Reprod. 2003; 18(1): 117-121, doi: 10.1093/humrep/deg016, indexed in Pubmed: 12525451.

11. Hui SK, Kapatoes J, Fowler J, et al. Feasibility study of helical tomotherapy for total body or total marrow irradiation. Med Phys. 2005; 32(10): 32143224, doi: 10.1118/1.2044428, indexed in Pubmed: 16279075.

12. Feygelman V, Opp D, Javedan K, et al. Evaluation of a 3D diode array dosimeter for helical tomotherapy delivery QA. Med Dosim. 2010; 35(4):324-329, doi: 10.1016/j.meddos.2009.10.007, indexed in Pubmed: 20097061.

13. Wong JYC, Filippi AR, Dabaja BS, et al. Total Body Irradiation: Guidelines from the International Lymphoma Radiation Oncology Group (ILROG). Int J Radiat Oncol Biol Phys. 2018; 101(3): 521-529, doi: 10.1016/j.ijrobp.2018.04.071, indexed in Pubmed: 29893272.

14. Litoborska J, Piotrowski T, Malicki J. Evaluation of three VMAT-TMI planning methods to find an appropriate balance between plan complexity and the resulting dose distribution. Phys Med. 2020 [Epub ahead of print]; 75: 26-32, doi: 10.1016/j.ejmp.2020.05.018, indexed in Pubmed: 32480353.

15. Vriesendorp HM. Aims of conditioning. Exp Hematol. 2003; 31(10): 844-854, doi: 10.1016/s0301-472x(03)00229-7, indexed in Pubmed: 14550799.

16. Van Dyk J, Galvin JM, Glasgow GP, Podgorsak EB. The Physical Aspects of Total and Half Body Photon Irradiation: A Report of Task Group 29 Radiation Therapy Committee. American Association of Physicists in Medicine, New York 1986. 